\title{
Chromosomal Investigations from Short-term Cultures of Embryonal Liver Cells
}

\author{
M. Macek \\ Institute of Child Development Research, Pediatric Faculty, Charles \\ University. Prague, Czechoslovakia
}

Received February 14, 1966

\begin{abstract}
Short-term cultivation of lymphoid cells from blood has for its simplicity and easy availability become the basic method of human cytogenetics (Moorhead et al. 1960). Shortterm cultivation of lymphoid cells of spleen, lymphatic glands and thymus (Baker and Atkin 1963) made possible a chromosomal investigation also after the death of the child and even from the foetuses from the 5 th month of intrauterine life onwards. It was not possible to make earlier a successful chromosomal investigation from short-term cultures of these organs (Bains and Gauld 1964). In view of the fact that in the early phases of embryonal development a greater number of chromosomally conditioned malformations (Carr 1964) may be expected, we tried to find out whether a chromosomal investigation from short-term cultures of cells obtained from embryonal liver, would be possible.
\end{abstract}

\section{Material and methods}

Short-term tissue cultures from embyronal liver cells were cultivated from 12 foetuses, 812 weeks old, which were obtained at artificial interruption of pregnancy mostly of young women who for different reasons did not desire pregnancy.

From the material obtained at interruption, liver or whole foetuses were taken under sterile conditions and put into the cultivation medium with antibiotics (penicilline and streptomycine). The tissue was rinsed in the laboratory several times in fresh medium and after preparation the liver tissue was cut into small fragments in three Petri dishes with the cultivation medium. The medium which was made turbid by the released cells was sucked off together with the fragments and divided into the cultivation flasks, type Legroux, volume 100 $\mathrm{ml}$ and completed up to $10 \mathrm{ml}$. Afterwards phytohaemagglutinine $\mathrm{M}$ (DIFCO) $0.3 \mathrm{ml} / 10 \mathrm{ml}$ medium was added. The cells were cultivated in the medium EPL (Michl 1961) without serum, $\mathrm{pH}$ adjusted by $\mathrm{NaHCO}_{3}$ according to the colour of the indicator to 7.4 .

For chromosomal investigation we used the fractionated blockade of the mitoses by colcemide (Macek 1965). To the 1 st flask colcemide was added at the 48 th hour of cultivation for 18 hours, to the 2 nd bottle at the 66 th hour of cultivation for 6 hours, and to the 3rd bottle at the 72nd hour of cultivation for 18 hours. After the blockade, the cells were shaken off from the glass and together with the cells, already previously released into the medium, carefully sucked off without fragments and centrifugated. The suspension obtained was hypotonised by a mixture of 1 part medium and 3 parts water or the cells were hypotonised directly in the cultivation bottle by adding $30 \mathrm{ml}$ of distilled water. After hypotonisation which did not exceed $30^{\circ}$, the cells after centrifugation were fixed by the chilled mixture of 1 part acetic acid and 3 parts metanol, finely resuspended and after $30^{\prime}$ of fixation centrifugated. After sucking off of the fixation the cells were resuspended in several drops of $45 \%$ acetic acid and preparations were made at once or within 24 hours (Macek 1965). After dyeing with $2 \%$ orcein solution the chromosomal investigation was carried out.

The mitotic activity was evaluated using the mitotic index-MI (the number of mitoses in 1000 cells studied). 


\section{Results}

Besides the blood elements also liver tissue cells are released during cutting and cultivation. From the fragments firmly adhered to the glass, fibroblasts and epitheloid cells grew already after $\mathbf{2 4}$ hours which after decanting of the medium remained on the glass of the cultivation flask. since we investigated only the cells released into the medium without trypsinisation. The cultivation was in all cases, but one, successful.

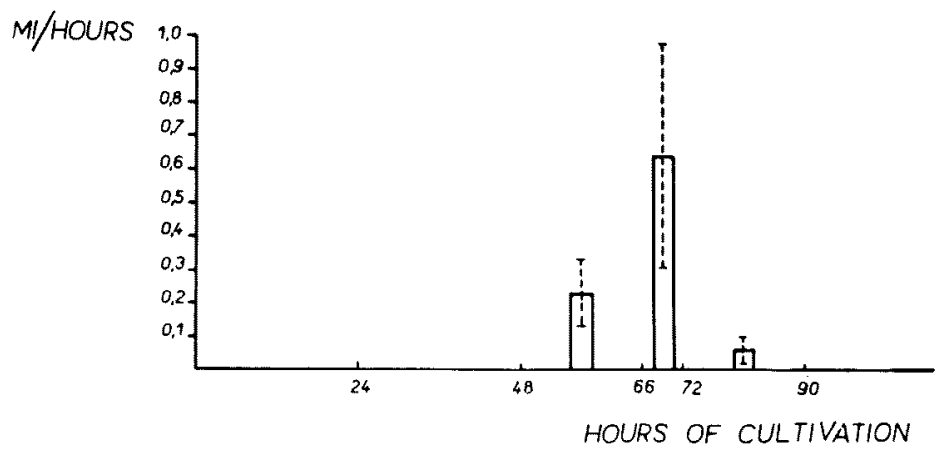

Fig. 1. Time development of mitotic activity. The mitotic activity is expressed in MI/hours, by its mean value for the Ist fraction/ 48th-66th hour/, 2nd fraction/66th-72nd hour/and for the 3rd fraction/72th-90th hour/and by a $95 \%$ confidence limit of the arithmetic means indicated.

The MI evaluation provided information about the mitotic activity in the individual fractions. In the first fraction (48th-66th hour) the mean value of MI was 4.18 , its lowest value 1.0 and the highest 9.0 . In the second fraction (66th-72nd hour of blockade) the mean value was 3.9. In the third fraction (72nd-90th hour of cultivation) it decreased to 1.1 , in 3 cases no mitoses were found in this fraction, in 2 cases the value of MI was 0.5 , whereby none of the ascertained values exceeded 3.0. After the 90th hour in the course of further three days of cultivation no mitoses were found by analogue evaluation.

The individual values obtained by MI were divided for individual fractions by the duration of the blockade (MI/hour) in order to find out in which fraction the mitotic activity is the highest. We concluded from statistical analysis that the mitotic activity differs significantly (mutually) in individual fractions and reaches the highest values in the 2 nd fraction as revealed by Fig. 1 . There the values of arithmetic means (MI) are indicated together with their 95\% confidence limit.

The results of the chromosomal investigation illustrated in Table 1 show that it was possible to obtain from the cultures a sufficient amount of mitoses for evaluation, mostly from the 1 st and 2 nd fraction, because the 3 rd fraction contained the smallest number of mitoses as can be seen also from the course of mitotic activity. We see from Table 1 that in all cases the basic number of 46 chromosomes was found. The number of polyploid mitoses did not exceed $4 \%$, there were hypotetraploid, tetraploid mitoses and in one case (Nr. 8) one octoploid mitosis was found.

With the use of the above-mentioned method the preparations from embryonal cells did not differ in quality from the material obtained from short-term cultures 


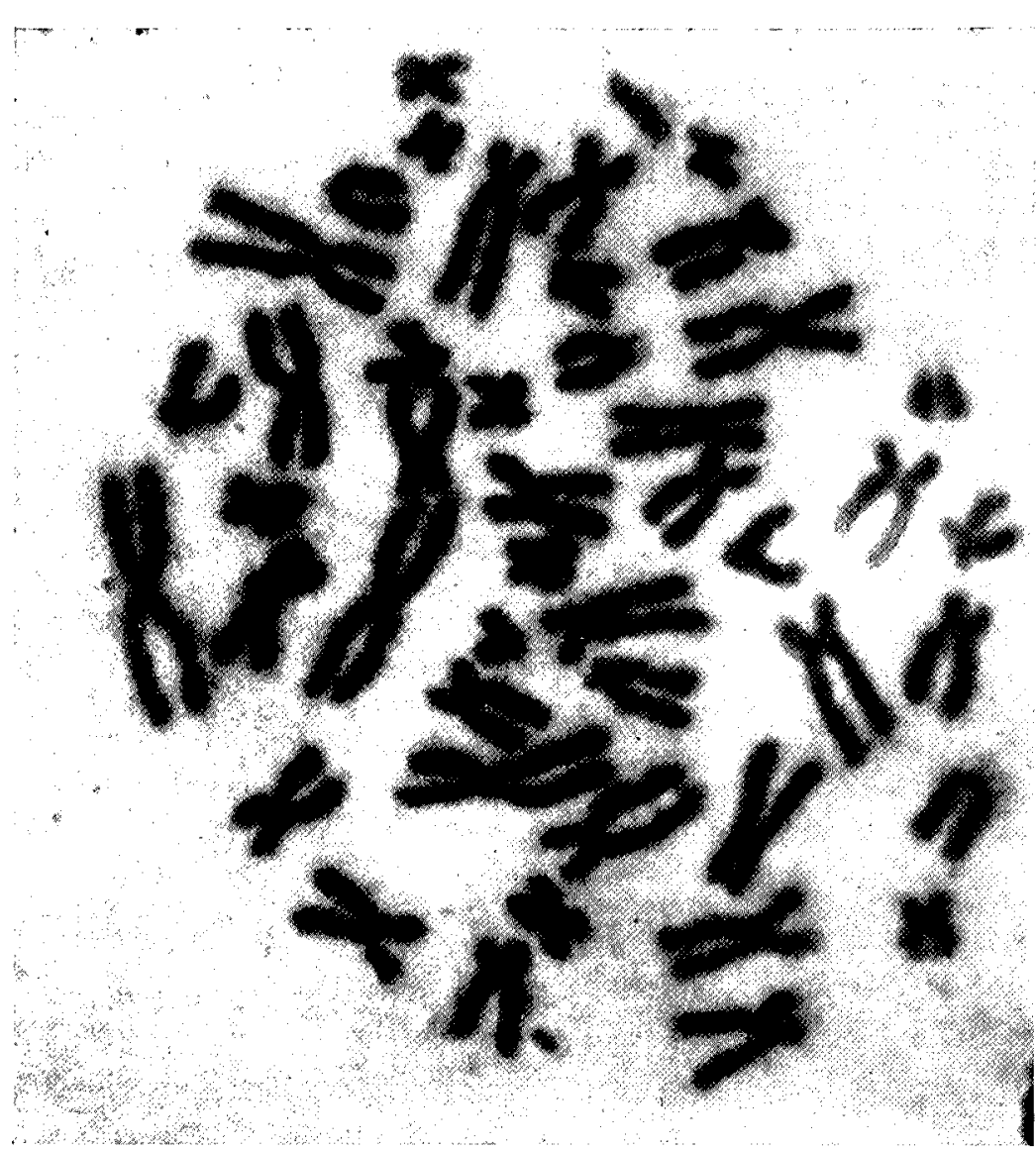

Fig. 2. Mitosis from the 1st fraction of a case Nr. 6. of peripheral blood. Fig. 2 shows a mitosis, Fig. 3 its karyotype (from the 1st fraction, case Nr. 6.).

We succeeded to sample the spleen (several millimeter size) from three 3 month old embryos. Similarly as in the liver, we proceeded with the short-term cultures. In one case the result of cultivation was negative, from the second case we obtained 11 mitoses which could be evaluated and in the third case 61 mitoses. The highest activity was also here in the 2nd fraction (MI 9.0$10.0)$.

\section{Discussion}

The above-desribed method of initiation of cultures makes possible to analyse simultaneously a higher number of samples in a short time, whereby cells are not lost by centrifugation, because all freed cells are

cultivated during the cutting and in addition we use the cells which are also released from liver fragments in the course of cultivation.

We consider it an advantage that with this kind of short-term cultivation it 
is not neces-

sary to use serum, similarly as is the case with cultivation of peripheral

blood leuko-
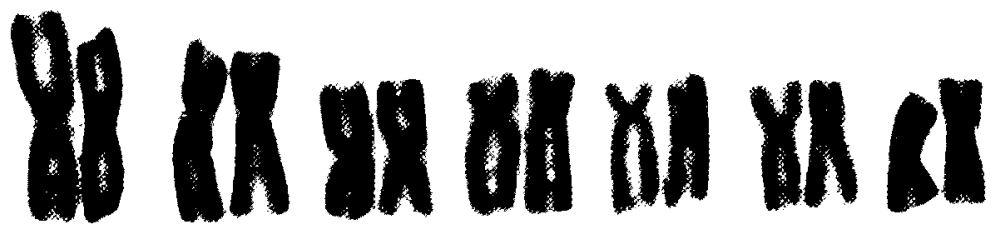

cytes (Macek 1965) or longterm cultivation of fibro-
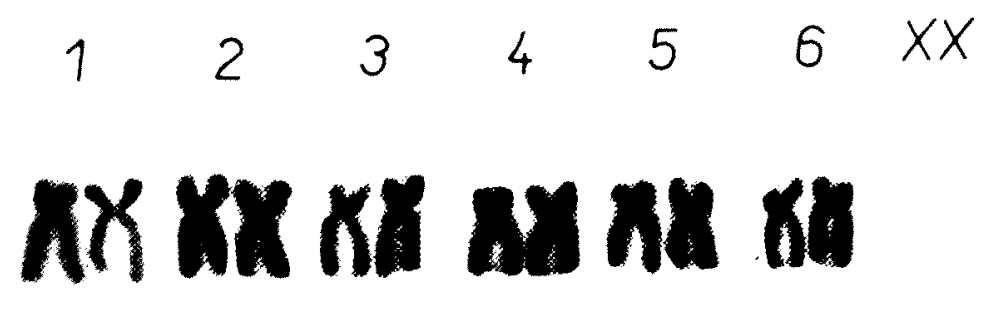

blast strains from embryo-
7
8
9
$10 \quad 11$
12

nal tissues

(Macek and Michl 1964).

The frac-
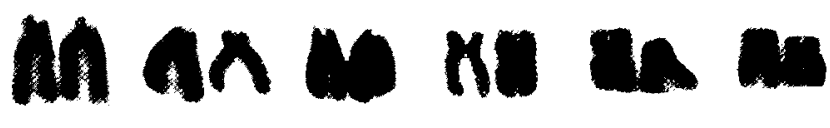

tionated blockade proved also here use$\begin{array}{llllll}13 & 14 & 15 & 16 & 17 & 18\end{array}$ ful, similarly as with cultivation of peripheral blood, since with the current use of blockade, our
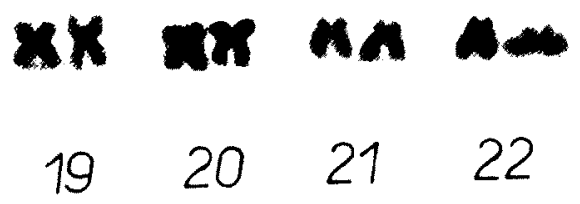
investigation would be after 72 hours cultivation unsuccessful also in those cases where we obtain a sufficient amount of mitoses for investigation from the 1st and 2 nd fraction. The reason for the lower values of MI compared with the cultivation of peripheral blood is also due to the fact that in contrast to the evaluation of MI from peripheral blood we count here not only blood cells (of hematopoetic tissues) but also cells released from liver tissue.

We suppose that the main source of mitoses in our cultures from this period of embryonal development are erythopoesis in liver sinusoides, erythrocytes from circulating blood which multiply actively during this period and to a lesser extent lymphocytes which appear in a greater amount only at the end of the third month of intrauterine life (Willis 1962). From our results we conclude that the adopted procedure is suitable for chromosomal investigation of short-term cultivation of cells, obtained from the liver, and thus to shift the boundary of this type of 
cultivation to the 2 nd or 3 rd month of intrauterine life.

A similar mode of cultivation and investigation is also possible in this period of development from the spleen but its importance for cytogenetics is greatly limited to the small amount of material which might be sufficient for other studies.

The amount of polyploid cells, though greater, than it is found in cultures from peripheral blood, does not exceed the limit observed in human diploid cell strains (Hayflick and Moorhead 1961). This can be the reflex of higher cell activity (Bain and Gauld 1961) or the manifestation of a multiplication of other cells as for example megakryocytes or cells from liver parenchyma.

\section{Summary}

1. A simple method of cultivation and chromosomal investigation of shortterm cultures of cells from embyronal liver is described which allows a successful chromosomal investigation already from the 2 nd-3rd month of intrauterine life.

2. From the analysis of the time course of the mitotic activity it resulted that its maximum is before the 72nd hour of cultivation. Afterwards the activity decreases significantly and after the 90 th hour of cultivation it disappears completely.

3. The chromosomal investigation did not prove a greater amount of chromosomal aberrations, though in the majority of cultures, polyploid cells were in $1-3 \%$ of mitoses; endoreduplication was found in one case only.

\section{Acknowledgement}

I wish to express my thanks to Mrs. Véra Zatrapová for her technical assistance.

\section{Literature}

Moorhead, P.S., Nowell, P.C., Mellman, W.J., Battips, D.M. and Hungerford, D.A. 1960. Exptl. Cell. Res. 20: 613-616.

Baker, C.M. and Atkin, N.B. 1963. Lancet 1: 1164.

Bain, A.D. and Gauld, S.K. 1964. Brit. J. Exptl. Path. 45: 530-532

Carr, D.H. 1963. Lancet 2: 603-606.

Michl, J. 1961. Exptl. Cell. Res, 25: 324-334.

Macek, M. 1965. Folia Biologica 11: 299-304.

Macek, M., Michl, J. 1964. Acta Univ. Carol. Medica 10: 519-539.

Willis, R.A. 1962. The Borderland of Embryology and Pathology. 2 ed., Butterworth, London. Haytlick, L. and Moorhead, P.S. 1961. Exptl. Cell Res. 25: 585-621.

Bain, A.D. and Gauld, J.K. 1964. Lancet 1: 936-937. 